\title{
Shared Challenges and Solutions: The Common Future of Comparative Politics and Quantitative Methodology
}

\author{
Xun Pang ${ }^{1,2}$
}

Received: 23 March 2016/Accepted: 18 June 2016/Published online: 20 July 2016

(C) Fudan University and Springer Science+Business Media Singapore 2016

\begin{abstract}
This essay joins the discussion on "The Future of Comparative Politics" from a perspective of methodology, and argues that the challenges concerned in Schmitter's essay are not endemic to comparative politics but shared ones in other research fields including quantitative methods. Recent trends and developments in quantitative methods show that quantitative and qualitative methods are increasingly integrated to jointly handle challenges with broad and profound impacts on the social sciences as a whole. This essay presents a brief introduction of the recent three revolutions in quantitative methods. The "Bayesian Revolution", the "Credibility Revolution", and the "Big Data Revolution" have fundamentally changed quantitative methods. The paper further displays that the challenges arising from the three revolutions are essentially the same ones with those in comparative politics, such as modeling complex interdependence, dealing with fuzzy concepts and the messy real world, and so on. Finally, the essay uses a few examples of some new analytical tools developed by quantitative methodologists to illustrate that qualitative knowledge and quantitative techniques should be seamlessly mixed to be innovative and powerful methods. All this points to a common future of comparative politics and quantitative methods.
\end{abstract}

Keywords Comparative politics · Quantitative methods · Complex interdependence · Globalization · Interaction

Xun Pang

xpang@tsinghua.edu.cn

1 School of Social Sciences, Tsinghua University, 314 Mingzhai Hall, 100084 Beijing, China

2 Institute for International Relations, Tsinghua University, Beijing, China 


\section{Introduction}

In the great essay, entitled "The Future of Comparative Politics: Western, Eastern or Both?" Philippe Schmitter investigates the profound challenges that comparativists face today, and presents insightful thoughts about how to deal with those challenges for a better future of comparative politics. Phil's article finds an echo in my heart, and I feel myself a comparativist when reading it, though I am "officially" a quantitative methodologist with International Relations as my major substantive research field. Epistemologically speaking, everyone is a comparativist because, as Phil put it, "comparison is an analytical method-probably the best available onefor advancing valid and cumulative knowledge about politics". Science is built on comparison. Nothing can be learnt without comparison. As one of the approaches to pursue scientific inquires, quantitative methods make descriptive or causal inference by comparing a relatively large number of observations. The discussion about the future of comparative politics is appealing to methodologists also because quantitative methods, as a research field, is driven by methodological problems when analyzing empirical data, and the sources for methodological innovations are exactly the challenges faced by scholars in substantive research fields.

Today, the field of quantitative methods is very different from decades ago, and its foreseeable future will be even more different. The field has experienced at least three revolutions in the past 30 years, being reshaped by profound and overwhelming challenges. ${ }^{1}$ Similar to comparative politics, the field of quantitative methods is also at a crossroad, and methodologists are debating whether the revolutionary trends are conflicting with one another and which of the possible paths should we take. ${ }^{2}$ It is extremely important for the two fields to have a dialog and exchange thoughts about the implications of, and possible solutions to, the challenges they commonly face. My understanding is that most issues Phil and many other comparativists are concerned about are essentially methodological, and they are by no means endemic to comparative politics but shared by other research fields, quantitative methods included. Quantitative methodologists have made a great effort to revise existing methods and invent new methods stimulated by the recent revolutions. However, the challenges are so profound that isolated endeavor is suboptimal and inefficient. Interdisciplinary and diverse approaches should be innovatively integrated to produce better solutions.

In this essay, I first would like to summarize how the three recent revolutions in the field of quantitative methodology pose challenges similar to the ones to comparativists. Then I present some examples of methodological solutions that may have potentials to assist comparativists to deal with their challenges. Those

\footnotetext{
${ }^{1}$ Latest innovations of quantitative methods by political methodology are published in political science journals, such as Political Analysis, The Journal of Politics, American Political Science Review, the American Journal of Political Science, and also increasingly appears in leading statistical journals, such as the Journal of American Statistical Association, Journal of the Royal Statistical Society, Journal of Computational and Graphical Statistics, and relatively young journals focusing more on unconventional methods, such as Methodological Innovations, Big Data Journal, Computational Social Networks.

${ }^{2}$ In 2005 the journal, Political Science \& Politics, published a symposium on the recent trends of quantitative methods.
} 
examples illustrate why development and implementation of innovative quantitative solutions should and have to rely heavily on qualitative knowledge and expert judgments. I would like to argue that, to tackle the shared challenges, it is counterproductive to stick to the conventional demarcation lines of quantitative and qualitative methods. The best solutions would come from merging, rather than simply combining, the two types of methods. Therefore, the two fields actually share one common future.

\section{Three Revolutions in Quantitative Methods}

To many, quantitative methods are more or less equivalent to econometrical regression models and statistical tests. Linear least squares regression is the modeling workhorse, and statistical inference relies on asymptotic theory by imagining infinitely future incoming observations. There have been many strong criticisms from the statistical community against inference relying on asymptotic, such as Barnett (1973); Berger et al. (1997), and Gelman et al. (1995). With real data and particular research goals, the linear assumption is often undefendable, then a variety of link functions and data transformation tricks were invented to linearize the world. The conventional practice of implementing quantitative methods is to choose the "best" standard model, and asymptotic properties of an estimator are automatically applied to accept or reject a tentative theory, even when the theory and data structure are not "standard" and the sample size is ridiculously small for the " $N$ goes to infinity" discussion. It is no surprise that sophisticated researchers in substantive fields often complain about quantitative methods for over-simplification and ignorance of the real world. Fortunately, this practice is already outdated, and cannot reflect the state of the art and the dynamic of the field. Since the 1990s, quantitative methods have experienced three revolutions that have dramatically changed the field.

\subsection{Bayesian Revolution: Free Modeling Complexity from Estimation Concerns}

The first among the three recent revolutions is the Bayesian revolution, driven by the rediscovery of Markov Chain Monte Carlo methods (Casella and George 1992; Gelman et al. 1995; Gill 2007). The Bayesian paradigm of statistical inference is anything but revolutionarily new. The Bayesian epistemology and Bayesian model setup are arguably even older than the frequentist paradigm of statistics. The Bayesian approach is originated from the Bayes theorem, and its model setup is quite mechanic as the following:

$$
\pi(\theta \mid y)=\frac{\pi(\theta) f(y \mid \theta)}{f(y)}
$$

This is also called Bayesian updating - to update our prior understanding of $\theta$ from $\pi(\theta)$ to the posterior, $\pi(\theta \mid y)$, with new observed empirical information $y$. 
The "Bayesian revolution" occured roughly around the early 1990s, thanks to the re-discovery of Markov Chain Monte Carlo methods (MCMC) (Metropolis and Ulam 1949; Hastings 1970; Gelfand and Smith 1990; Casella and George 1992; Chib and Greenberg 1995). After half a centaury since they were invented, statisticians finally realized how powerful the two basic MCMC sampling schemes, namely, the Gibbs sampler and the more general Metropolis-Hastings algorithm. Statisticians then quickly figured out the theoretical and mathematical foundations of MCMC. This is revolutionary to statistics and its practitioners because MCMC enables specifications of statistical models according to what research needs rather than what conventional estimating techniques allow. More precisely, the Bayesian model setup and MCMC techniques end the situation that the researcher had to tailor her theory in order to fit a standard or known statistical model. Instead, the researcher can now specify parameter-rich models with structures as complicated as her theory requires. The conventional standard models resort to strong assumptions and simplification to make the model to be linear or generalized linear, because they need to have a closed-form solution of the estimator and analytically develop their asymptotic properties. Refer to almost all textbooks of applied statistics or econometrics for such a template of presenting a model: a standard model specification, a list of statistical assumptions, mathematical expressions of the estimator, development and presentation of unbiasedness and asymptotic properties of the estimator. Refer to the most popular econometrics textbooks, such as Wooldridge (2001). If the researcher chooses to apply "quantitative methods", she has to accept the unrealistic assumptions or the level of simplification of a certain model (such as linear models, poison models, various panel data estimators, to name but a few). It appeared as an unavoidable trade-off between implementing quantitative methods and having a complex theory to be empirically tested. ${ }^{3}$ Powered by MCMC, Bayesian models free scientific social theories from strict technical constraints and revolutionarily change the way that empirical research could be done (Refer to Robert (2007)).

\subsection{Credibility Revolution: Identify Causation with Subject-Matter Knowledge}

The second revolution is the credibility revolution that broke out in political science roughly at the turn of the New Millennium. It is "a movement that emphasizes the goal of obtaining secure causal inferences (Clark and Golder 2015 p. 65)." Conventionally, ceteris paribus is the essence of causation, and it is also how regression results are interpreted: in a linear regression, a causal effect is expressed as "holding all other things constant", and in a nonlinear one, this is done with a minor modification as "holding all other variables at their mean (median, minimum, maximum, or other) values". When a researcher routinely uses such thinking and expressions, she might have not even thought about whether it was even logically possible to hold some of those variables constant while letting the variable of

\footnotetext{
${ }^{3}$ Thanks to Phil Schmitter for suggesting the "non-linear revolution" which is the core part in the Bayesian revolution.
} 
primary interest to vary. In the credibility revolution, causality is re-defined in the "potential outcomes" language and counterfactual thinking. It is frankly admitted, "data reveal only associations, which are a combination of causal and non-causal (i.e., spurious) components (Keel, 2015, p. 102)." Causation is identified with assumptions or conditions under which observable associations can be reasonably regarded as reflecting causal effects. Mathematically speaking, we need assumptions to achieve the following equation:

$$
\operatorname{Pr}(Y \mid X=x)=\operatorname{Pr}\left(Y^{X=x}\right) \text { or } E(Y \mid X=x)=E\left(Y^{X=x}\right)
$$

In the equations, the conditional probability (or the conditional expectation) on the left-hand side is a statistical expression of association between $Y$ and $X$, and the right-hand side term is the probability (or expectation of) a potential outcome of $Y$ given a value of the treatment $X$, which is defined as causation. For more detailed explanation, refer to some of excellent books on causal inference, such as Hernán and Robins (2001).

The acceptance of the fact that causation can only be identified with assumptions rather than modeling techniques, at least for observational studies, has profound implications to quantitative methods. It is recognized that data analysis is not enough to estimate causal effects, no matter how advanced computational techniques are and how big the sample size is. Instead, identification of causal effects in observational studies has to resort to unquantifiable subject-matter knowledge and expert judgments. For instance, the graphic theory of causality was invented and has been widely applied by statisticians, but the theory is not quantitative but qualitative (Pearl 1995). Causal diagrams can only be drawn with deep knowledge about the subject under investigation, and the selection of confounders depends on experts' judgments on how things are interconnected in the real world. The concept of identification in the credibility revolution is different from identification in statistics. The latter is to ensure the uniqueness of underlying data-generation processes, and can be achieved and checked without resorting to qualitative knowledge. Identification in the credibility revolution is about the conditions under which we can regard one group of observations as counterfactuals of another group. Because counterfactuals are not observable, causality can only be identified by assumptions and judged by experts.

\subsection{Big Data Revolution: Discover Patterns from Messiness}

In recent years, big data has been one of the hottest buzzwords across all industries as well as in academia. What does the "Big Data" revolution mean to the scientific community is still unclear, and scientists in different disciplines hold different, often opposite, views toward big data and about how the science community should react to it. Radical views, often from business circles, computer and data sciences, argue that big data fundamentally changes the definition and practice of science. They claim that "correlation is enough", predict the end of theory, and advocate that "scientists no longer have to make educated guesses, construct hypotheses and models, and test them with data-based experiments and examples (Anderson 2008; 
Prensky 2009)." But most researchers in natural and social sciences fully recognize the profound implications of big data but do not think we should go as far as letting "giant computers to do science".

The volume, structure, and velocity of social data demand researchers to revise the conventional tools and invent new methods. Big data are not only "big", but also high-dimensional, relational, and messy. Big data can better reflect the complex and dynamic real world. They are different from conventional "small data" in terms of how data are generated, collected, stored, analyzed, and utilized. In the past, quantitative methods were tools to handle "cleaned" data, and "elegant" simple models were enough to understand the simplified world reflected by the highly aggregated data. But much of the big data are social data, meaning that they are "fat" (high-dimensional) and "relational" (interdependent). They are "data about the interactions of people or their collectivities: how they communicate, how they form relationships, how they come into conflict, and how they shape their future interactions through political and economic institutions (Monroe et al. 2015, p. 71)." In "The Future of Comparative Politics", Phi points out that one of the critical challenges to comparative politics is a violation of the assumption of independent cases. This assumption is critical for scientific comparison, but is too restrictive in the era of globalization and complex interdependence. With increasing availability of relational data, quantitative methods are faced with the same challenge - one of the most fundamental assumptions in regression analysis is the i.i.d. assumption, (observations are independent random draws from an identical distribution, conditional on the observables), but it can no longer be held.

The big data revolution changes the priority of scientific inquiries, too. Causal inference has long been regarded as the most "noble" and ultimate goal of scientific research. It may still be. But with complex data, description is now a much more difficult and important research task. The conventional view is that description is necessary but simple, as preparation for causal inference. This is clearly demonstrated by the relative unimportant tables of descriptive statistics and correlation matrices of variables in research articles. Now we have big data from the noisy and messy real world, and interdependence makes it impossible to use simple dimension reduction by projecting a high-dimension space onto a one-dimensional variable. With theoretical and technical challenges of big data, quantitative methods are much more serious about description and pattern discovery than before. The fast-growing research areas of automated information extraction, opinion mining, and network analysis, are primarily for discovering patterns but not analyzing causal relations between simple variables (Lazer et al. 2009; Cioffi-Revilla 2014).

\section{Shared Challenges: Comparative Politics and Quantitative Methodology}

The three revolutions sequentially occurred and jointly created new research tasks that have profoundly changed the field of quantitative methods. The Bayesian revolution frees model setup from rigid technical constraints, and enables the researcher to develop and test theories with complex and rich structures. The 
credibility revolution requires quantitative (statistical) inference of causal effects to rely more explicitly on comparison of potential outcomes or counterfactuals, and causality can only be identified and justified with subject-matter knowledge. The big data revolution means that quantitative methods should serve as tools for more diverse research tasks and assist the researcher to observe, discover, explore, and describe the complex and messy world. The challenges to comparative politics stated in the essay of Phil Schmitter are shared by quantitative methods.

\subsection{Globalization, Interdependence, and Complexity}

Simplification is necessary for doing science. All theories and methods do simplification in a certain degree in order to understand the real world. There is nothing wrong to simplify, and the real question lies in what is the proper degree of simplification. Simplification is a serious research task, but not an excuse for seeking elegance of theories or models at the expense of neglecting the complexity of the real world.

Complex interdependence is a straight violation of crucial assumptions on which the conventional statistical models are based. Whenever the i.i.d. assumption is violated, the conventional practice is to correct the standard errors to justify the desired properties of an estimator. One may easily find econometric textbooks and research articles for a large number of error correction tricks. The widely applied PSCE approach in political science is an easy, simple, and all-in-one correction of interdependence, as proposed in Beck and Katz (1995).

In other words, the conventional quantitative methods regard interdependence as nuisance to be corrected for, but not a phenomenon of research interest, even though many theories and hypotheses focus on the causes or consequence of complex interdependence in the era of globalization (Franzese and Hays 2008; Franzese et al. 2012; Chaudoin et al. 2015).

Another challenge of interdependence to quantitative methods is that interdependence means high-dimensional modeling. Without the i.i.d. assumption, we have to deal with an $n$-dimensional multivariate random variable. A change in any dimension has to be analyzed with a simultaneous consideration of changes in all other dimensions. "The curse of dimensionalities" is well known to quantitative analysts, and dimension reduction is a difficult research task with complex interdependence. One of the conventional (probably the easiest) ways of dimension reduction is to rely on the i.i.d. assumption to reduce an n-dimensional joint distribution into $\mathrm{n}$ independent one-dimensional distributions. However, with the increasing importance of interdependence in substantive research, quantitative methods have to straightly face "the curse of dimensionalities" and develop techniques to systematically model, rather than simply correct for, the "statistical problems" caused by interdependence.

Phil Schmitter further points out that another serious challenge of complexity interdependence is that complexity violates the assumption of effect stability in comparative political research. This is also a challenge to quantitative methods. Regression models assume constant coefficients across units and time and deny the possibility of context-dependent relations between variables. Parametric 
quantitative models such as $E(Y)=f(X, \theta)$, requires assumptions about $f($.$) which$ plays a crucial role to ensure a closed-form solution of the estimator, about the number of parameters, $\theta$, that has to be small enough to guarantee enough degrees of freedom; and about potential explanatory variables, $X \mathrm{~s}$, many of which are zeroed out often with implicit assumptions. But complexity requires a much more complicated $f($.$) than a linear or concave function required for OLS or MLE to have$ closed-form solutions. A population with heterogeneous components causes another type of complication-multimodal functions and mixture distributions should be used to summarize the population, and group-specific parameters are necessary to capture different causal mechanisms in different sub-populations. In addition, complexity also means the system composes of more actors and factors, and many $X$ s cannot be easily zeroed out to get a simple system.

From a perspective of methodology, complex interdependence means heterogeneous actors (individuals and collectivities) at different levels (relatively defined as micro and macro levels) make decisions and take actions while expecting, observing, and reacting to, how others act. The trade-off between accuracy and parsimony can no longer be guided with statistical rules, such as what is recommended in King et al. (1994, p. 74):

$$
\operatorname{MSE}(g)=V(g)+E(g-\gamma) 2=\text { variance }+ \text { Squared bias }
$$

Instead, how to balance the trade-off should be decided by what are the interpretable and meaningful results a researcher can get when she studies a world of complex interdependence. Reduction and isolation are necessary to make sense of the world. Without reduction and isolation, we cannot summarize and interpret what we observe. But, how much the high-dimensional real world can be reduced to a low-dimensional theoretical world? How small a part of the world can be isolated from the rest of the world? If the isolated low-dimensional world no longer represents the real world, then nothing can be learnt about the real world, no matter how well the estimating procedure is conducted.

\subsection{Variables, Fuzzy Concepts, and Patterns}

Quantitative methods are based on probability theory and work with random variables. Broad acceptance and implementation of quantitative methods have popularized the statistical terminology, for instance, the statistical term "variables" is used everyday in many fields. Phil Schmitter argues, "comparative politics has tended to focus on variables". But in my opinion, qualitative methods actually focus on "concepts" instead of variables, and here "variable" is used as an exchangeable to "concept". Before a study is quantified, a "variable" is simply a concept. Variables are used to investigate probabilities of occurrence of events. We do not care about variables per se, but we are interested in their characteristics and relations with other variables, such as the domain, range, defining parameters, density function, probabilities, and correlation coefficients with other variables. At the same time, a variable can be univariate or multivariate, and the distribution function of a variable can have a few, a lot, or even infinitely many parameters. Variables are not 
necessarily "simple" or standard ones taught in stats textbooks. Rather, they can be as complicated as the researcher can imagine. Monte Carlo simulation methods are developed to empirically study the characteristics of complex variables (Christian and Casella 2007). Variables are quantities to express uncertainty about anything of research interest, such as concepts, relationships, patterns, and the truth (in the Bayesian paradigm) (Jaynes 2003). A researcher can define her own variables and apply simulation techniques to know the characteristics of a nonstandard and complicated variable.

To quantitative methods, the new challenge is how to use variables to express concepts and patterns. Variables, concepts, and patterns are three different things existing in three different worlds - variables are statistical quantities, concepts are theoretical entities, and patterns are empirical beings. A scientific study needs to connect the three worlds. Without transforming concepts into variables, no statistical tools can be applied. Without representing concepts, variables and their realizations (data) are meaningless or even misleading in substantive research, and quantitative methods can generate insensible results. Moreover, patterns can inspire new concepts via inductive reasoning, and can give ideas about what distributional assumptions could be made as well as what variance-covariance matrix could be specified for a multivariate distribution.

The real challenge to both quantitative methods and comparative politics is not to choose which one among concepts, patterns, and variables, but how to connect the three and transform them into one another. Conventionally, quantitative methods are applied after conceptualization and operationalization of concepts have been done, while comparativists proceed as if they have to force fuzzy and multidimensional concepts into simple variables in order to apply quantitative methods. Quantitative analysts know more about how to specify a variable to capture the fuzziness, uncertainty, and dimensionality of a concept, but they need to work with people in the substantive fields to understand the concepts and their theoretical applications.

\subsection{Merge Qualitative and Quantitative Methods}

"Bridge the gap" between quantitative and qualitative methods, has been often advocated in the concluding section in essays discussing about advantages or disadvantages of the two types methods. But many in each "camp" still see the two types of methods are rivalries to each other. Phil Schmitter observed that more and more researchers use both quantitative methods and a small $\mathrm{N}$ analysis of carefully selected cases, and so did I and many others. I am happy to see that more scholars now choose methods according to their research tasks, disregarding the "ideological lines". But I think it could be even more productive and powerful to "merge" the quantitative and qualitative methods. The task is challenging but extremely promising.

The aforementioned three revolutions jointly point to the indispensability of "merging" qualitative knowledge and quantitative analysis. As mentioned above, the credibility revolution elevates the importance of qualitative knowledge in causality identification in observational studies. Identification of casual effects requires qualitative methods, and then estimation of causal effects is conducted with 
quantitative methods. And the two tasks are integral in causal inference, and qualitative and quantitative methods are merged in a single method of causal inference.

The Bayesian revolution requires to specify priors. To some people, priors are the unscientific component of the Bayesian approach, because the information in priors does not come from "objective" data but from subjective knowledge from the researcher. At the same time, they regard priors as a necessary evil to use powerful MCMC techniques, and try to minimize the influence of priors on the posteriors to let the objective likelihood (data) to completely dominate Bayesian learning. Recognizing the value of unquantifiable information and knowledge, many others advocate to embrace the opportunity provided by Bayesian setup and use priors to incorporate relevant information we have before we see new data. Priors can and should be specified with existing theoretical judgments, expert knowledge, and even intuitions of the researcher (Greenberg, 2008). Priors fully representing our existing understandings and knowledge are valuable and helpful for advancing the cumulative process of learning. Furthermore, the Bayesian approach, with prior specification, explicitly requires that the researcher be an expert of a subject before she analyzes the data. If a researcher is completely ignorant about the subject, then she is not qualified to conduct the research, no matter how many data are collected or how skillful she is as a data analyst. Like the credibility revolution, the Bayesian revolution merges qualitative and quantitative methods in a single process of Bayesian updating.

The big data revolution blurs the lines between quantitative and qualitative methods in a more fundamental way. Most big data are not numeric data, but texts, audios, videos, images, etc. They are often unstructured and require qualitative judgments to make sense of the data and extract signals form noise. Pattern discovery is one of the major tasks in big data analysis and inspires new developments of quantitative methods. In turn, qualitative researchers collect data that are increasingly "big" in their volume and velocity, and information extraction and data analysis require quantitative techniques. Therefore, if the conventional distinction between quantitative and qualitative methods depends on whether data are quantified or not, the big data revolution erases the distinction all together. If the defining difference between quantitative and qualitative methods is the way of information processing, machine and human approaches are both needed in any data analysis in the big data era and the two types of methods are merged by big data.

\section{Joint Solutions and Common Future}

In the last three decades, the field of quantitative methods has been changed and advanced through dealing with the challenges brought by three revolutions. There are numerous new tools invented for doing diverse research tasks. In this section, I would like to use some important recent developments in quantitative methods as examples to show that new tools are increasingly available to deal with the challenges, and to argue that better solutions to the shared problems come from a joint effort of comparativists and quantitative methodologists. 


\subsection{More Careful Comparison for Causal Inference}

The field of comparative politics is named with its primary method of comparison, and the credibility revolution redefines causal effects as a comparison of "potential outcomes" or counterfactuals (Lewis 1973; Rubin 1974; Holland 1986; Rubin 1986). The research task of identification and the tools for implementing identification, such as various matching approaches or re-sampling approaches, make it clear that, in pursuing casual studies, quantitative methods share the same methodological foundation of comparative case studies (Imbens and Angrist 1994; King and Zeng 2006; Iacus et al. 2011; Ho et al. 2007; Imai et al. 2009). The only difference is that quantitative methods are primarily interested in average causal effects of many matched cases, and the comparative case study is interested more in individual casual effects of a few matched cases. Comparativists are good at doing careful selection of cases, presenting justifications of case selections, and carefully comparing cases for causal inference. Their trainings, expertise, skills, and experiences are precious for causal inference pursuing average causal effects with large number of observations.

Many political scientists, comparativists included, do field experiments, especially survey experiments. Is this a qualitative or a quantitative way of doing research? The design of an experiment needs statistical training and local knowledge, and requires quantitative methodologists and comparativists to work together to figure out survey questions, sampling schemes (randomization or stratification), potential confounders, solutions to missing data, and so on. What is the "treatment?" What is the population? Are the control group and treatment group exchangeable? These types of questions are not simply statistical or substantive questions.

As mentioned before, another powerful tool for identifying causation is graphical theory (Pearl, 2011). Graphical theory is a statistical invention, but is not a "quantitative" tool. Graphical theory is used to find confounders, i.e., what are the factors that must be controlled for in order to identify a casual effect of $X$ on $Y$. In causal inference, variable selection is far more "qualitative" than the conventionally-applied econometric rules, such as the rule that variables, $\{Z: \operatorname{cor}(Z, X) \neq 0$, and $\operatorname{cor}(Z, Y) \neq 0\}$, should be controlled for to have an unbiased estimator. Graphical diagrams are drawn to help investigate what other association relations should be blocked to draw the conclusion that the observed association between $X$ and $Y$ is equal to the causal effect. The justification of what variables should be included to block back door paths (confounding association) is mainly based on subject-matter knowledge.

Furthermore, I doubt that identification of causal effects, even with perfect randomization, can satisfy the curiosity of working scientists about causal mechanisms or questions about "why?" No matter whether we rely on conventional regression models to achieve ceteris paribus, or adopt counterfactual (potential outcomes) comparison, quantitative methods and causal inference designs are still silent about causal mechanisms (Cox 2000, p. 424). Mediation analysis in the causal inference literature claims that it investigates the causal mechanism (Imai et al. 2010). But mediation analysis is still about testing causal mechanisms but not 
explaining "why". A causal story can only be constructed in a qualitative way, and the curiosity for causal explanations can only be satisfied with qualitative methods, such as in-depth narratives and process tracing.

The recent advancement of quantitative methods on causal inference provides solid methodological rules for testing hypothesized causal relations, but without qualitative knowledge and analysis, we cannot obtain causal inferences or explanations.

\subsection{Modeling Complex Interdependence}

As comparativists try to tackle issues arising from complex interdependence, quantitative methodologists are developing new tools to accommodate complexity and interdependence of the real world. The Bayesian revolution enables the researcher to specify sophisticated models. MCMC makes high-dimensional integration an easy job to do, and now we can model interdependence among a large number of units. Cross-level interactions can be nicely incorporated in Bayesian multilevel modeling. In addition, the flexibility of Bayesian model setup and powerful MCMC tools imply that scholars can analyze horizontal and hierarchical interdependence in a single model. The big data revolution has stimulated many inventions of new analytical methods to model complexity and interdependence, such as network analysis and complex social modeling. The rapid advancement of quantitative methods highlights the crucial role of qualitative knowledge. I may illustrate this point with a few examples.

Spatial modeling might be one of the most familiar methods to political scientists for modeling interdependence (Franzese and Hays 2007, 2008). Spatial econometrics has been criticized for the pre-specified spatial metric $W$, i.e., the big spatial matrix containing important information about the pattern or structure of interconnections among all units (Pang 2014). Different specifications of the spatial metric can generate very different inferences of all parameters in the model, not only the spatial autoregressive coefficient associated to W. Critics are worried about the lack of robustness of spatial modeling, and are unconformable about the fact that the W matrix is specified with qualitative knowledge (Arbia and Fingleton 2008; Conley 1999; Conley and Topa 2002). Indeed, specification of the $W$ matrix injects important information into the model, and only experts of the subject might be able to specify and justify the $W$ matrix with their qualitative judgments about interdependence. Quantitative methods can estimate the effect of interdependence, but qualitative knowledge is necessary to know the structure of interdependence. The solution has to rely on both.

The second example is network analysis, which has been increasingly applied in political science to study interdependence (Hafner-Burton and Montgomery 2009; Ward et al. 2011; Maoz 2010). Spatial modeling can be regarded as a special case of network analysis. Network analysis is a descriptive tool to summarize characteristics of a system and units as well as the features of relationships (ties) connecting units. Quantitative methods can be used for data reduction, classifying cliques, calculating numeric expressions of systemic or individual features, estimating trends of network evolution and inter-network dependence. But qualitative methods and 
knowledge is essential for constructing a network by answering the theoretical questions of what is a network? Why is such a network interesting? What are the characteristics of a network that should explain or be explained?

The third example is Bayesian multilevel modeling for analyzing cross-level interaction (Gelman and Hill 2006; de Leeuw and Meijer 2008). One of the advantages of the Bayesian model setup is its flexibility. A statistical model can be specified to closely reflect complex theory and reality. More flexibility does not imply less disciplined model specification. Rather, flexibility means that statistical model specifications require more expert judgments. For instance, a Bayesian multilevel model with two levels can be expressed as the following:

$$
\begin{gathered}
E\left(y_{j[i]}\right)=f\left(X_{j[i]}, \theta_{j}\right),(\text { level } 1) \\
E\left(\theta_{j}\right)=g\left(Z_{j}, \alpha\right),(\text { level } 2)
\end{gathered}
$$

Although this is a very simple expression of a multilevel model, it shows that the flexibility of model specification requires serious qualitative judgments. For instance, what group $j$ should an observation $i$ belong to? What coefficients among the $\theta$ s should be constant or varying across groups? What are the contextual factors $Z_{j}$ that can explain the variation of causal effects, $\theta_{j}$ s, across groups? Are two levels enough to capture the hierarchical structure of the data and serve well enough for the research purposes? Less rigid constraints on model specification require more cautious choices and more educated guesses.

\subsection{Discovery, Description, and Measurement}

In social sciences, many important concepts are fuzzy, comprising multiple layers of attributes (Collier and Seawright 2012; Collier and Levitsky 2009; Munck and Verkuilen 2002). It may be impossible to precisely describe and measure a concept in an abstract and general sense, but I believe that, in any particular research, it is still necessary to define, describe, and measure a concept as precisely as we can.

The field of quantitative methods has fully recognized the vital roles of description and measurement, and been trying to develop tools and methods to better describe and measure the fuzziness and complexity of social concepts. Quantitative methods used to regard themselves as tools for hypothesis testing, and only enter a substantive research after the theory and hypotheses have been developed and formatted. Where the theory and hypotheses come from is not a concern of quantitative tools. Quantitative methods are for theory-testing but not theory-discovery. But today, at the frontier of quantitative methods are inventions of new approaches of discovery, description, and measurement. From a perspective of methodology, all the three tasks are about data reduction-with high-dimensional space and relationships reflected by data, what reduction we can properly achieve to make sense of the data and the real world.

One of the most cited examples of quantitative achievements for measurement tasks in political science might be the VoteView project and the NOMINATE scores. Political ideology of an individual or a political collectivity is anything but a 
simple variable. A political position is a high-dimensional and fuzzy concept, and it cannot be directly observed. Quantitative methods use information aggregation with roll call data and dimension reduction to project the multidimensional political position to a left-right (liberal-conservative) spectrum. Measurement models, such as the Item Response Theory Model, have successfully located political positions in the low-dimensional latent policy space.

Another example is the measure of power. Power is one of the most important concepts in political science. It is a rational and context-dependent concept by nature (Baldwin 2013; Haugaard 2012; Hart 1976; Dahl 1957). Scholars have tried for a long time to measure power, but most of the measures are far from satisfactory and even invalid (Singer and Small 1972; Beckman 1984; Organski and Kugler 1980; Kugler and Domke 1986; Alcock and Newcombe 1970; German 1960; Barnett and Duvall 2005). Scholars fully realized the invalidity of most measures of power, and some even claimed that the concept of power is so fuzzy and contextcontingent that it is immeasurable (Guzzini 2005, 2009). That is to say, power cannot be regarded as a variable since it cannot be systemically described and empirically measured. But the recent development of network analysis provides a new way to define and measure the concept of power. By taking into account the structure of interactions rather than focusing on monadic characteristics of a unit, power can be measured with different types of centrality scores. And different types of centrality reflect different types of power, for instance, power originated from being a hub, being a bridge, from a credible threat of exit, and so on (Hafner-Burton and Montgomery 2009). With transparent definitions of power and rules of data reduction, a researcher can choose the most relevant one according to her particular research tasks.

Operationalization and measurement of concepts in one research may be irrelevant in another. Definition, description, and measure of a concept in social sciences are not context-free but depend on particular research questions and tasks. Challenges of description and discovery can be successfully tackled with qualitative knowledge, data analytical tools, and scientific research designs.

\section{Conclusion: The Common Future}

In the essay "The Future of Comparative Politics", Phil Schimitter expresses his preference that the future of comparative politics should follow the direction of "embrace complexity". This is also the direction that I see the field of quantitative methods is advancing. Today, quantitative methods are more diverse and versatile than in the past. The future of quantitative methods will be less like a distinct research field, but be more integrated with substantive research inquires into an emerging discipline called Computational Social Science, where statistical tools, computational skills, and qualitative knowledge and judgments are applied to explore, describe, explain, and solve social issues arising from the complicatedly interdependent world (Lazer et al. 2009). Comparative politics and Quantitative methods share similar challenges, demand joint solutions, and have a common future. 
Acknowledgments I would like to thank all participants for their helpful comments at the "Workshop on the Future of Comparative Politics in Conjunction with Fifth Year Anniversary Ceremony of CCPDS", held at Fudan University, May 14-15, 2016, Shanghai, China. This research is supported by Tsinghua University Initiative Scientific Research Program.

\section{References}

Alcock, Norman Z, and Alan G. Newcombe. 1970. The perception of national power. Journal of Conflict Resolution 14:335-343.

Anderson, C. 2008. The end of theory: the data deluge makes the scientific method obsolete. http:// archive.wired.com/science/discoveries/magazine/16-07/pb_theory.

Arbia, G., and B. Fingleton. 2008. New spatial econometric techniques and applications in regional science. Regional Science 87(3): 311-317.

Baldwin, David A. 2013. Power and International Relations. In Handbook of International Relations, 2 ed. Walter Carlsnaes, Thomas Risse, and Beth A. Simmon, 273-297. Thousand Oaks: SAGE Publications.

Barnett, Vic. 1973. Comparative statistical inference. New York: Wiley.

Barnett, Michael, and Raymond Duvall. 2005. Power in international politics. International Organization 59(01): 39-75.

Beckman, Peter. 1984. World politics in the twentieth centuty. Englewood Cliffs, NJ: Prentice-Hall.

Beck, Nathaniel, and Jonathan N. Katz. 1995. What to do (and not to do) with time- series cross-section data. The American Political Science Review 89(3): 634-647.

Berger, James O., B. Boukai, and Y. Wang. 1997. Unified frequentist and Bayesian testing of a precise hypothesis. Statistical Science 12: 133-160.

Casella, George, and Edward I. George. 1992. Explaining the Gibbs sampler. The American Statistician 46(3): 167-174.

Chib, Siddhartha, and Edward Greenberg. 1995. Understanding the Metropolis-Hastings Algorithm. The American Statistician 49(4): 327-335.

Christian, Robert P., and George Casella. 2007. Monte Carlo statistical methods. New York: Springer.

Cioffi-Revilla, Claudio. 2014. Introduction to computational social science: principles and applications. New York: Springer.

Chaudoin, Stephen, Helen V. Milner, and Xun Pang. 2015. International systems and domestic politics: linking complex theories with empirical models in international relations. International Organization 69(2): 275-309.

Clifford German. 1960. A tentative evaluation of world power. Journal of Conflict Resolution 4:138-144.

Clark, William Roberts and Matt Golder. 2015. Big data, causal inference, and formal theory: contradictory trends in political science. Political Science and Politics. pp. 65-70.

Collier, David, Jody LaPorte, and Jason Seawright. 2012. Putting typologies to work: concept-formation, measurement, and analytic rigor. Political Research Quarterly 65(1): 217-232.

Collier, David, and Steven Levitsky. 2009. Democracy conceptual hierarchies in comparative research. In Concepts and method in the social science: the tradition of Giovanni Sartori, ed. David Collier, and John Gerring. London: Routledge.

Conley, T.G. 1999. GMM estimation with cross sectional dependence. Journal of Econometrics 92: 1-45.

Conley, T.G., and G. Topa. 2002. Socio-economic distance and spatial patterns in unemployment. Journal of Applied Econometrics 17: 303-327.

Cox, D.R. 2000. Comment on 'causal inference without counterfactuals'. Journal of the American Statistical Association 96(450): 424-425.

Dahl, Robert A. 1957. The concept of power. Behavioral Science 2(3): 201-215.

de Leeuw, Jan, and Erik Meijer. 2008. Handbook of Multilevel Analysis. New York: Springer.

Franzese, Robert J., and Jude C. Hays. 2008. Interdependence in comparative politics. Comparative Political Studies 41(4-5): 742-780.

Franzese, R.J., and J.C. Hays. 2007. Spatial econometric models of cross-sectional interdependence in political science panel and time-series-cross-section data. Political Analysis 15: 140-164.

Franzese, Robert J., Jude C. Hays, and Aya Kachi. 2012. Modeling history dependence in networkbehavior coevolution. Political Analysis 20(2): 175-190. 
Gelfand, Alan, and Adrian F.M. Smith. 1990. Sampling-based approaches to calculating marginal densities. Journal of the American Statistical Association 85(410): 398-409.

Gelman, Andrew, John B. Carlin, Hal S. Stern, and Donald B. Rubin. 1995. Bayesian data analysis. New York: Chapman and Hall.

Gelman, Andrew, and Jennifer Hill. 2006. Data analysis using regression and multilevel/hierarchical models. New York: Cambridge University Press.

Gill, Jeff. 2007. Bayesian methods: a social and behavioral sciences approach. Boca Raton: Chapman and Hall CRC.

Greenberg, Edward. 2008. Introduction to Bayesian econometrics. New York: Cambridge University Press.

Guzzini, Stefano. 2005. The concept of power: A constructivist analysis. Millennium-Journal of International Studies 33(3):495-521.

Guzzini, Stefano. 2009. On the measure of power and the power of measure in international relations. DIIS working paper 28:1-18.

Hafner-Burton, Miles Kahler, and Alexander H. Montgomery. 2009. Network analysis for international relations. International Organization 63(3): 559-592.

Hart, Jeffrey. 1976. Three approaches to the measurement of power in international relations. International Organization 30(2): 289-305.

Hastings, W.K. 1970. Monte Carlo sampling methods using Markov Chains and their applications. Biometrika 55(1): 97-109.

Haugaard, Mark. 2012. Rethinking the four dimensions of power. Journal of Political Power 5(1): 35-54.

Hernán, Miguel A., and James M. Robins. 2001. Causal Inference. New York: CRC Press.

Ho, Daniel E., Kosuke Imai, Gary King, and Elizabeth A. Stuart. 2007. Matching as nonparametric preprocessing for reducing model dependence in parametric causal inference. Political Analysis 15(3): 199-236.

Holland, P.W. 1986. Statistics and causal inference. Journal of the American Statistical Association 81: 945-970.

Iacus, Stefano M., Gary King, and Giuseppe Porro. 2011. Multivariate matching methods that are monotonic imbalance bounding. Journal of the American Statistical Association 493(106): 345-361.

Imai, Kosuke, Gary King, and Clayton Nall. 2009. The essential role of pair matching in clusterrandomized experiments, with application to the Mexican Universal Health Insurance Evaluation (with discussions and rejoinder). Statistical Science 24(1): 29-53.

Imai, Kosuke, Luke Keele, and Dustin Tinley. 2010. A general approach to causal mediation analysis. Psychological Methods 15(4): 309-334.

Imbens, Guido W., and Joshua D. Angrist. 1994. Identification and estimation of local average treatment effects. Econometrica 62(2): 467-476.

Jaynes, E. T. 2003. Probability Theory: The Logic of Science. Cambridge University Press.

Keel, Luke. 2015. The Discipline of Identification. Political Science and Politics. pp. 102-105.

King, Gary, Robert O. Keohane, and Sidney Verba. 1994. Designing social inquiry: scientific inference in qualitative research. Princeton: Princeton University Press.

King, Gary, and Langche Zeng. 2006. The dangers of extreme counterfactuals. Political Analysis 14: 131-159.

Kugler, Jacek, and William Domke. 1986. Comparing the strength of nations. Comparative Political Studies 19:39-69.

Lazer, David, Alex Pentland, Lada Adamic, Sinan Aral, Albert-László Barabási, Devon Brewer, Nicholas Christakis, Noshir Contractor, James Fowler, Myron Gutmann, Tony Jebara, Gary King, Michael Macy, Deb Roy, and Marshall Van Alstyne. 2009. Computational social science. Science 323: 721-733.

Lewis, D.K. 1973. Counterfactuals. Oxford: Balckwell.

Maoz, Zeev. 2010. Networks of nations: the evolution, structure, and impact of international networks. New York: Cambridge University Press.

Metropolis, Nicholas, and S. Ulam. 1949. The Monte Carlo method. Journal of the American Statistical Association 44(247): 335-341.

Monroe, Burt L., Jennifer Pan, Margaret E. Roberts, Maya Sen, and Betsy Sinclair. 2015. No! formal theory, causal inference, and big data are not contradictory trend in political science. Political Science and Politics 48: 71-74.

Munck, Gerardo I., and Jay Verkuilen. 2002. Conceptualizing and measuring democracy evaluating alternative indices. Comparative Political Studies 35(1): 5-34. 
Organski, F.K., and Jacek Kugler. 1980. The war ledger. Chicago: The University of Chicago Press.

Pang, Xun. 2014. Varying responses to common shocks and complex cross-sectional dependence: dynamic multilevel modeling with multifactor error structures for time-series cross-sectional data. Political Analysis 22(4): 464-496.

Pearl, Judea. 1995. Causal diagrams for empirical research (with discussion). Biometrika 82(4): 669-710.

Pearl, Judea. 2011. Causality: models, reasoning, and inference. Cambridge: Cambridge University Press.

Prensky, Marc. 2009. "From digital immigrants and digital natives to digital wisdom." http://www. innovateonline.info/index.php?view14article\&id14705.

Robert, Christian P. 2007. The Bayesian core. New York: Springer.

Rubin, D.B. 1974. Estimating causal effects of treatment in randomized and nonrandomized studies. Journal of Educational Psychology 66: 688-701.

Rubin, D.B. 1986. Which ifs have causal answers? (comment on "statistics and causal inference" by P.W. Holland). Journal of the American Statistical Association 81: 961-962.

Singer, David J., and Melvin Small. 1972. The wage of war, 1816-1965. A Statistical Handbook. New York: Wiley.

Ward, Michael D., Katherine Stovel, and Andrey Sacks. 2011. Network analysis and political science. Annual Review of Political Science 14: 245-264.

Wooldridge, Jeffrey M. 2001. Econometric analysis of cross section and panel data. Cambridge: MIT Press. 